\title{
AN ALTERNATIVE CHARACTERIZATION FOR MATRIX EXPONENTIAL DISTRIBUTIONS
}

\author{
MARK FACKRELL, ${ }^{*}$ University of Melbourne
}

\begin{abstract}
A necessary condition for a rational Laplace-Stieltjes transform to correspond to a matrix exponential distribution is that the pole of maximal real part is real and negative. Given a rational Laplace-Stieltjes transform with such a pole, we present a method to determine whether or not the numerator polynomial admits a transform that corresponds to a matrix exponential distribution. The method relies on the minimization of a continuous function of one variable over the nonnegative real numbers. Using this approach, we give an alternative characterization for all matrix exponential distributions of order three.
\end{abstract}

Keywords: Matrix exponential distribution; rational Laplace-Stieltjes transform

2000 Mathematics Subject Classification: Primary 60E10

Secondary 62E10

\section{Introduction}

Matrix exponential (ME) distributions were first introduced in 1955 by Cox [9], [10] as distributions with rational Laplace-Stieltjes transform (LST). Lipsky and Ramaswami [18] (see also [2]) showed that such distributions can also be defined in terms of a generator matrix $\boldsymbol{T}$, a row vector $\boldsymbol{\alpha}$, and a column vector $\boldsymbol{t}$, rather like the familiar phase-type distributions introduced by Neuts [20] (see also [21, Chapter 2]) but without the simple probabilistic interpretation in terms of Markov chains. Asmussen and O'Cinneide [4] provided an excellent introduction to the topic.

Although ME distributions have been used in many diverse areas such as queueing theory [8, pp. 321-331], [17], [26], insurance risk [1, pp. 240-244], [5], renewal theory [2], [7], and telecommunications [16], [19], their usage is significantly less widespread than that of phasetype distributions. There are two main reasons for this. First, there is no simple probabilistic interpretation for ME distributions; and second, there is no straightforward method to ascertain whether or not a given generator $\boldsymbol{T}$, and vectors $\boldsymbol{\alpha}$ and $\boldsymbol{t}$ correspond to an ME distribution. In this paper we focus on the second problem; excellent interpretations for ME distributions can be found in [3] and [7].

There are only a few instances in the literature where the second problem has been addressed, and then only for particular classes of ME distributions; see, for example, [15], [27], [28], and [29]. Dehon and Latouche [11] approached the problem of characterizing generalized hyper-exponential distributions geometrically, as did O'Cinneide [24], who used the concept of invariant polytopes (see [23]).

Fackrell [12] (see also [6]) developed a geometric characterization for ME distributions. A necessary condition for a rational LST to correspond to an ME distribution is that its pole of maximal real part is both real and negative (see [28]). If this is the case then the set

Received 3 April 2007; revision received 7 April 2009.

* Postal address: Department of Mathematics and Statistics, University of Melbourne, Victoria 3010, Australia.

Email address: fackrell@unimelb.edu.au 
of all admissible numerator coefficients can be defined in terms of an uncountably infinite intersection of linear half-spaces. Using this characterization, Fackrell [12] gave a complete analytic description for the set (called $\Omega_{p}, p$ being the order of the ME representation) for the order 3 case. In this paper, rather than attempt to derive an analytic description for $\Omega_{p}$ in general, we focus on determining when a given rational LST corresponds to an ME distribution. In fact, this approach enables us to develop an alternative description for $\Omega_{3}$.

The structure of the paper is as follows. In Section 2 we formally define ME distributions. The development of $\Omega_{p}$ is described in Section 3. In Section 4 we present our algorithm to determine, given a suitable denominator polynomial, whether or not a rational LST's numerator polynomial corresponds to an ME distribution. Section 5 contains some examples to illustrate the approach. The alternative characterization for $\Omega_{3}$ is given in Section 6 , and the paper concludes with Section 7.

\section{Matrix exponential distributions}

A nonnegative random variable is distributed according to an ME distribution if its distribution function, defined for $u \geq 0$, has the form

$$
F(u)= \begin{cases}\alpha_{0}, & u=0 \\ 1+\alpha \exp (\boldsymbol{T} u) \boldsymbol{T}^{-1} \boldsymbol{t}, & u>0\end{cases}
$$

where, for finite $p \geq 1, \boldsymbol{\alpha}$ is a $1 \times p$ row vector, $\boldsymbol{T}$ is a $p \times p$ matrix, and $\boldsymbol{t}$ is a $p \times 1$ column vector, all possibly with complex entries. The parameter $\alpha_{0} \in[0,1]$ is known as the point mass at zero and can be derived from the other parameters. We will not consider the case when $\alpha_{0}=1$ as this gives the trivial distribution function. Distribution (2.1) is said to have a representation $(\boldsymbol{\alpha}, \boldsymbol{T}, \boldsymbol{t})$ of order $p$. The corresponding density function, defined for $u>0$, is given by

$$
f(u)=\boldsymbol{\alpha} \exp (\boldsymbol{T} u) \boldsymbol{t} .
$$

We set $f(0)=\boldsymbol{\alpha} \boldsymbol{t}$ for convenience. The LST of (2.1), defined for at least $s$ in the right complex plane and possibly for $s$ with $\operatorname{Re}(s)>-\delta$ for some positive number $\delta$, is given by

$$
\begin{aligned}
\phi(s) & =\int_{0}^{\infty} \mathrm{e}^{-s u} \mathrm{~d} F(u) \\
& =\boldsymbol{\alpha}(s \boldsymbol{I}-\boldsymbol{T})^{-1} \boldsymbol{t}+\alpha_{0} \\
& =\frac{a_{p} s^{p-1}+a_{p-1} s^{p-2}+\cdots+a_{1}}{s^{p}+b_{p} s^{p-1}+b_{p-1} s^{p-2}+\cdots+b_{1}}+\alpha_{0},
\end{aligned}
$$

where $a_{1}, a_{2}, \ldots, a_{p}, b_{1}, b_{2}, \ldots, b_{p}$ are all real; see [2] or [18]. The zeros of the numerator polynomial $a(s)$ are referred to as the zeros of the LST, and the zeros of the denominator polynomial $b(s)$ are referred to as the poles of the LST. If $a(s)$ and $b(s)$ have no factors in common then the algebraic degree of the ME distribution is defined to be the degree of $b(s)$; see [22].

In general, representations for ME distributions are not unique, and they do not necessarily have the same order. A representation that has minimal order will be called a minimal representation. The order of the ME distribution is defined to be the order of any minimal representation. 
Given a rational LST of the form (2.2) that corresponds to an ME distribution, we can easily find an ME representation for it. One such representation is given by

$$
\begin{aligned}
\boldsymbol{\alpha} & =\left(a_{1}, a_{2}, \ldots, a_{p}\right) \\
\boldsymbol{T} & =\left(\begin{array}{cccccc}
0 & 1 & 0 & \ldots & 0 & 0 \\
0 & 0 & 1 & \ldots & 0 & 0 \\
0 & 0 & 0 & \ddots & 0 & 0 \\
\vdots & \vdots & \ddots & \ddots & \ddots & \vdots \\
0 & 0 & 0 & \ddots & 0 & 1 \\
-b_{1} & -b_{2} & -b_{3} & \ldots & -b_{p-1} & -b_{p}
\end{array}\right), \\
\boldsymbol{t} & =\left(\begin{array}{c}
0 \\
0 \\
\vdots \\
0 \\
1
\end{array}\right)=\boldsymbol{e}_{p} ;
\end{aligned}
$$

see [2, Proposition 2.3]. Note that this representation is not necessarily minimal.

The representation (2.3)-(2.5) is important for a number of reasons. First, it shows that a representation with only real parameters can be found for any ME distribution. Second, because the characteristic equation of the companion matrix (2.4) is

$$
b(s)=s^{p}+b_{p} s^{p-1}+b_{p-1} s^{p-2}+\cdots+b_{1}=0
$$

the set of eigenvalues of $\boldsymbol{T}$ contains the set of poles of the LST (the two sets will be identical if $a(s)$ and $b(s)$ have no factors in common). Third, and most importantly, every ME distribution has a unique minimal representation of the form (2.3)-(2.5), whose order is the distribution's algebraic degree. If this is the case then the corresponding $a(s)$ and $b(s)$ will have no factors in common.

Owing to the one-to-one correspondence between the polynomials $a(s)$ and $b(s)$, and the representation (2.3)-(2.5), for the remainder of the paper, we will freely interchange between the polynomials, representation, and the vectors $\boldsymbol{a}=\left(a_{1}, a_{2}, \ldots, a_{p}\right)$ and $\boldsymbol{b}=\left(b_{1}, b_{2}, \ldots, b_{p}\right)$.

\section{Characterization of matrix exponential distributions}

In this section we describe the development of $\Omega_{p}$, the set of all vectors $\boldsymbol{a}$ that admit ME distributions, as long as $b(s)$ has a zero of maximal real part that is real and negative. For the remainder of the paper, we assume that $b(s)$ satisfies this condition.

For $\boldsymbol{a}$ and $\boldsymbol{b}$ to correspond to an ME distribution,

1. $f(u)=\boldsymbol{\alpha} \exp (\boldsymbol{T} u) \boldsymbol{e}_{p} \geq 0$ for $u \geq 0$, and

2. $0<a_{1} / b_{1} \leq 1$. 
Suppose that condition 2 holds. Then condition 1 imposes constraints on $a_{2}, a_{3}, \ldots, a_{p-1}$. Let

$$
\exp (\boldsymbol{T} u) \boldsymbol{e}_{p}=\left(\begin{array}{c}
f_{1}(u) \\
f_{2}(u) \\
\vdots \\
f_{p}(u)
\end{array}\right)
$$

a $p \times 1$ vector of functions depending on $u$. It can be shown that, for $i=1,2, \ldots, p$,

$$
f_{i}(u)=f_{1}^{(i-1)}(u)=\boldsymbol{e}_{1}^{\top} \boldsymbol{T}^{i-1} \exp (\boldsymbol{T} u) \boldsymbol{e}_{p},
$$

where $f_{1}^{(i)}(u)$ is the $i$ th derivative of $f_{1}(u), \boldsymbol{e}_{i}$ is the $p \times 1$ vector with a 1 in the $i$ th position and 0 s elsewhere, and ' $T$, denotes the transpose. Furthermore,

$$
f_{1}(0)=f_{1}^{(1)}(0)=f_{1}^{(2)}(0)=\cdots=f_{1}^{(p-2)}(0)=0 \quad \text { and } \quad f_{1}^{(p-1)}(0)=1 ;
$$

see [12, Theorem 5.11] or [6].

Now, for $u \geq 0$, the inequality in condition 1 can be written as

$$
f(u)=a_{1} f_{1}(u)+a_{2} f_{2}(u)+\cdots+a_{p} f_{p}(u) \geq 0 .
$$

Since $a_{1}=b_{1}\left(1-\alpha_{0}\right)$ (see [12, Lemma 5.2] or [6]), there will be a different family of constraints for each $\alpha_{0} \in\left[0,1\right.$ ). The situation can be simplified by dividing (3.2) by $1-\alpha_{0}$ (which is positive), and letting, for $i=1,2, \ldots, p-1$,

$$
x_{i}=\frac{a_{i+1}}{1-\alpha_{0}},
$$

so that inequality (3.2) is expressed as

$$
b_{1} f_{1}(u)+x_{1} f_{2}(u)+\cdots+x_{p-1} f_{p}(u) \geq 0 .
$$

In order to satisfy condition 1 , we need to find constraints on $x_{1}, x_{2}, \ldots, x_{p-1}$ so that (3.3) holds for all $u \geq 0$.

Let

$$
\boldsymbol{x}=\left(x_{1}, x_{2}, \ldots, x_{p-1}\right) .
$$

Each $x \in \mathbb{R}^{p-1}$ corresponds to a function, defined for $u \geq 0$, of the form

$$
g(\boldsymbol{x}, u)=b_{1} f_{1}(u)+x_{1} f_{2}(u)+\cdots+x_{p-1} f_{p}(u) .
$$

Since we require that $g(\boldsymbol{x}, u) \geq 0$ for all $u \geq 0$, we need to find all $\boldsymbol{x} \in \mathbb{R}^{p-1}$ such that (3.3) holds for all $u \geq 0$. This defines a region in $\mathbb{R}^{p-1}$,

$$
\Omega_{p}=\bigcap_{u \geq 0}\left\{\boldsymbol{x} \in \mathbb{R}^{p-1} \mid b_{1} f_{1}(u)+\sum_{i=1}^{p-1} x_{i} f_{i+1}(u) \geq 0\right\} .
$$

The above argument implies that $f(u)=\left(1-\alpha_{0}\right) g(\boldsymbol{x}, u)$ is the density function of an ME distribution if and only if $x \in \Omega_{p}$. We note here that $\Omega_{p}$ is nonempty, closed, convex, and bounded; see [6, Theorem 3.1]. 
Given $\boldsymbol{a}$ and $\boldsymbol{b}$, it is relatively simple to check that the zero of maximal real part of $b(s)$ is real and negative, and condition 2 holds. Checking condition 1, that is, determining if $x \in \Omega_{p}$, is much more difficult. The description of $\Omega_{p}$ as the intersection of an uncountable number of linear half-spaces makes it difficult to determine whether a given point is contained in it or not. Nevertheless, Fackrell [12, Theorems 6.4 and 6.5] (see also [6, Theorems 4.3 and 4.4]) gave a complete description of $\Omega_{3}$, although the process was involved. We now give the description of $\Omega_{3}$, first when the zeros of $b(s)$ are all real, and then when two zeros are a complex conjugate pair.

Theorem 3.1. Let $\boldsymbol{b}=\left(b_{1}, b_{2}, b_{3}\right)$. Suppose that the zeros of $b(s)=s^{3}+b_{3} s^{2}+b_{2} s+b_{1}$, $-\lambda_{1},-\lambda_{2}$, and $-\lambda_{3}$, are real and such that $0<\lambda_{1} \leq \lambda_{2} \leq \lambda_{3}$. For $u \geq 0$, let $f_{1}(u)$ be defined by (3.1). Then the boundary of $\Omega_{3}, \partial \Omega_{3}$, consists of

1. the line segment between $(0,0)$ and $\left(\lambda_{2} \lambda_{3}, 0\right)$,

2. the line segment between $\left(\lambda_{2} \lambda_{3}, 0\right)$ and $\left(\lambda_{3}\left(\lambda_{1}+\lambda_{2}\right), \lambda_{3}\right)$, and

3. the parametric curve $\Sigma_{3}$, defined, for $u \geq 0$, by

$$
\begin{aligned}
& x_{1}(u)=b_{1} \frac{f_{1}^{(1)}(u) f_{1}^{(2)}(u)-f_{1}(u) f_{1}^{(3)}(u)}{f_{1}^{(1)}(u) f_{1}^{(3)}(u)-\left(f_{1}^{(2)}(u)\right)^{2}}, \\
& x_{2}(u)=b_{1} \frac{f_{1}(u) f_{1}^{(2)}(u)-\left(f_{1}^{(1)}(u)\right)^{2}}{f_{1}^{(1)}(u) f_{1}^{(3)}(u)-\left(f_{1}^{(2)}(u)\right)^{2}} .
\end{aligned}
$$

Theorem 3.2. Let $\boldsymbol{b}=\left(b_{1}, b_{2}, b_{3}\right)$. Suppose that the zeros of $b(s)=s^{3}+b_{3} s^{2}+b_{2} s+b_{1}$, $-\lambda_{1},-\lambda_{2}$, and $-\lambda_{3}$, are such that $\lambda_{1}=\mu, \lambda_{2}=\alpha+\mathrm{i} \beta$, and $\lambda_{3}=\alpha-\mathrm{i} \beta$ with $0<\mu \leq \alpha$ and $\beta>0$. For $u \geq 0$, let $f_{1}(u)$ be defined by (3.1). Let $\tilde{u}$ be the minimal positive solution to

$$
f_{1}(u) f_{1}^{(2)}(u)-\left(f_{1}^{(1)}(u)\right)^{2}=0 .
$$

Then $\partial \Omega_{3}$ consists of

1. the parametric curve $\Sigma_{3}$, defined, for $0 \leq u \leq \tilde{u}$, by

$$
\begin{aligned}
& x_{1}(u)=b_{1} \frac{f_{1}^{(1)}(u) f_{1}^{(2)}(u)-f_{1}(u) f_{1}^{(3)}(u)}{f_{1}^{(1)}(u) f_{1}^{(3)}(u)-\left(f_{1}^{(2)}(u)\right)^{2}}, \\
& x_{2}(u)=b_{1} \frac{f_{1}(u) f_{1}^{(2)}(u)-\left(f_{1}^{(1)}(u)\right)^{2}}{f_{1}^{(1)}(u) f_{1}^{(3)}(u)-\left(f_{1}^{(2)}(u)\right)^{2}},
\end{aligned}
$$

and

2. the line segment between $(0,0)$ and $\left(x_{1}(\tilde{u}), 0\right)$, where

$$
x_{1}(\tilde{u})=-b_{1} \frac{f_{1}(\tilde{u})}{f_{1}^{(1)}(\tilde{u})} .
$$

Figure 1 shows $\Omega_{3}$ when $b(s)=(s+1)(s+2)(s+3)$. The grey lines are the boundaries of some of the half-spaces defining $\Omega_{3}$, and the black lines and curve depict $\partial \Omega_{3}$. 


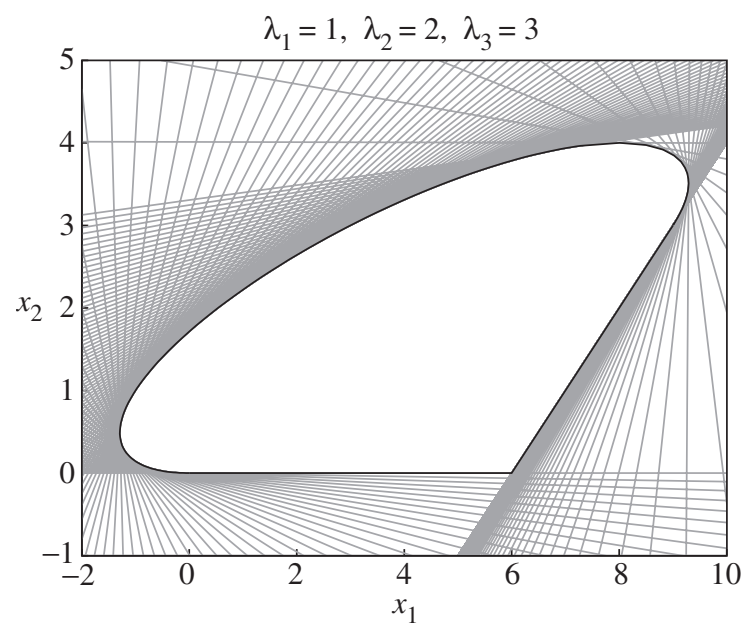

FIGURE 1: Plot of $\Omega_{3}$ and its boundary $\partial \Omega_{3}$.

\section{The matrix exponential identification algorithm}

In this section we will tackle the problem of determining if a given $\boldsymbol{x} \in \mathbb{R}^{p-1}$ belongs to $\Omega_{p}$ on a 'case-by-case' basis. That is, given $\boldsymbol{x} \in \mathbb{R}^{p-1}$, we develop an algorithm to determine whether or not $x \in \Omega_{p}$ without actually deriving a parametric description for the whole of $\partial \Omega_{p}$. In fact, for the case when $p=3$, this approach actually does lead us to a complete description of $\partial \Omega_{3}$, arrived at via an approach different to that in Section 3. The method we develop is related to a technique Dehon and Latouche [11] used to characterize the class of all generalized hyperexponential distributions of algebraic degree 3 .

Let $\boldsymbol{a}$ and $\boldsymbol{b}$ satisfy condition 2 in Section 3. Condition 1 is satisfied if and only if

$$
\hat{\boldsymbol{x}}=\left(\hat{x}_{1}, \hat{x}_{2}, \ldots, \hat{x}_{p-1}\right)=\frac{b_{1}}{a_{1}}\left(a_{2}, a_{3}, \ldots, a_{p}\right)
$$

is contained in the set $\Omega_{p}$.

Recall, from (3.4), that $\hat{\boldsymbol{x}}$ corresponds to the function, defined for $u \geq 0$,

$$
g(\hat{\boldsymbol{x}}, u)=b_{1} f_{1}(u)+\hat{x}_{1} f_{1}^{(1)}(u)+\hat{x}_{2} f_{1}^{(2)}(u)+\cdots+\hat{x}_{p-1} f_{1}^{(p-1)}(u) .
$$

In order to determine if $\hat{\boldsymbol{x}} \in \Omega_{p}$ (that is, corresponds to an ME distribution), we need to ascertain whether $g(\hat{\boldsymbol{x}}, u) \geq 0$ for all $u \geq 0$. Since we are discussing $\Omega_{p}$ from a geometrical point of view, in Figure 2, $\hat{\boldsymbol{x}}$ is depicted by the point $Q$ which has coordinates $\hat{\boldsymbol{x}}$.

Let $\overline{\boldsymbol{x}}=\left(\bar{x}_{1}, \bar{x}_{2}, \ldots, \bar{x}_{p-1}\right)$ correspond to the mixture of the point mass at zero and the exponential distribution

$$
F(u)= \begin{cases}\alpha_{0}, & u=0, \\ 1-\left(1-\alpha_{0}\right) \mathrm{e}^{-\lambda_{1} u}, & u>0,\end{cases}
$$

where $0 \leq \alpha_{0}<1$; see Figure 2. The vector $\overline{\boldsymbol{x}} \in \Omega_{p}$ since $g(\overline{\boldsymbol{x}}, u)=\lambda_{1} \mathrm{e}^{-\lambda_{1} u}>0$ for all $u \geq 0$. In Figure 2 we depict $\overline{\boldsymbol{x}}$ by the point $P$ which has coordinates $\overline{\boldsymbol{x}}$. Also, for $i=1,2, \ldots, p-1$, 


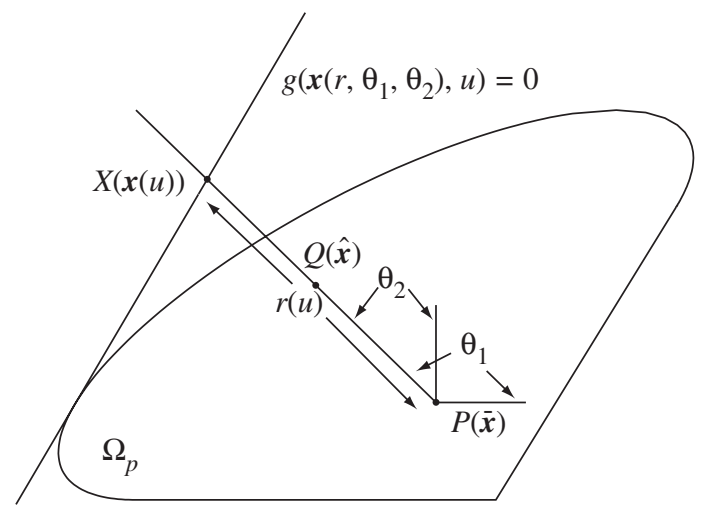

FigURE 2: Diagram of $\Omega_{3}$ showing the points $P(\overline{\boldsymbol{x}}), Q(\hat{\boldsymbol{x}})$, and $X(\boldsymbol{x}(u))$.

$\bar{x}_{i}$ is the coefficient of $s^{i}$ in the expansion of $\lambda_{1}\left(s+\lambda_{2}\right)\left(s+\lambda_{3}\right) \cdots\left(s+\lambda_{p}\right)$. This is the case because the LST of (4.2) can be expressed as

$$
\begin{aligned}
\phi(s) & =\frac{\left(1-\alpha_{0}\right) \lambda_{1}}{s+\lambda_{1}}+\alpha_{0} \\
& =\frac{\left(1-\alpha_{0}\right) \lambda_{1}\left(s+\lambda_{2}\right)\left(s+\lambda_{3}\right) \cdots\left(s+\lambda_{p}\right)}{\left(s+\lambda_{1}\right)\left(s+\lambda_{2}\right)\left(s+\lambda_{3}\right) \cdots\left(s+\lambda_{p}\right)}+\alpha_{0} \\
& =\frac{\left(1-\alpha_{0}\right)\left(\bar{x}_{p-1} s^{p-1}+\bar{x}_{p-2} s^{p-2}+\cdots+\bar{x}_{1} s+b_{1}\right)}{s^{p}+b_{p} s^{p-1}+b_{p-1} s^{p-2}+\cdots+b_{1}}+\alpha_{0} .
\end{aligned}
$$

Let $\overrightarrow{P Q}$ denote the ray emanating from $P$ (with coordinates $\overline{\boldsymbol{x}}$ ) and passing through $Q$ (with coordinates $\hat{\boldsymbol{x}}$ ). For $i=1,2, \ldots, p-1$, let $\theta_{i} \in[0, \pi]$ be the angle $\overrightarrow{P Q}$ makes with the ray emanating from $P$ in the direction parallel to the $i$ th positive coordinate axis. Let $\boldsymbol{\theta}=\left(\theta_{1}, \theta_{2}, \ldots, \theta_{p-1}\right)$. We have

$$
\cos \theta_{i}=\frac{\hat{x}_{i}-\bar{x}_{i}}{\|\hat{\boldsymbol{x}}-\overline{\boldsymbol{x}}\|_{2}},
$$

where $\|\cdot\|_{2}$ denotes the $L_{2}$-norm. Any point on the line through $P$ and $Q$, extending in both directions, has, for $i=1,2, \ldots, p-1$ and $r \in \mathbb{R}$, coordinates given by

$$
x_{i}\left(r, \theta_{i}\right)=\bar{x}_{i}+r \cos \theta_{i} .
$$

For $u \geq 0$, define $r(u)$ to be the $L_{2}$-distance from $P$ to the hyperplane

$$
g(\boldsymbol{x}, u)=b_{1} f_{1}(u)+x_{1} f_{1}^{(1)}(u)+x_{2} f_{1}^{(2)}(u)+\cdots+x_{p-1} f_{1}^{(p-1)}(u)=0,
$$

measured in the direction determined by the ray $\overrightarrow{P Q}$ (or $\boldsymbol{\theta}$ ). Suppose that, for any given $u \geq 0$, $\overrightarrow{P Q}$ meets the hyperplane defined by (4.7) at the point $X$ (which has coordinates $x(u)=$ $\left.\left(x_{i}(u), x_{2}(u), \ldots, x_{p-1}(u)\right)=\overline{\boldsymbol{x}}+r(u) \cos \boldsymbol{\theta}\right)$. Letting $r=r(u)$ in (4.6) and substituting into (4.7) gives

$$
b_{1} f_{1}(u)+\sum_{i=1}^{p-1}\left(\bar{x}_{i}+r(u) \cos \theta_{i}\right) f_{1}^{(i)}(u)=0
$$


Rearranging (4.8) gives

$$
\begin{aligned}
r(u) & =\frac{-b_{1} f_{1}(u)-\bar{x}_{1} f_{1}^{(1)}(u)-\bar{x}_{2} f_{1}^{(2)}(u)-\cdots-\bar{x}_{p-1} f_{1}^{(p-1)}(u)}{\cos \theta_{1} f_{1}^{(1)}(u)+\cos \theta_{2} f_{1}^{(2)}(u)+\cdots+\cos \theta_{p-1} f_{1}^{(p-1)}(u)} \\
& =\frac{-\lambda_{1} \mathrm{e}^{-\lambda_{1} u}}{\cos \theta_{1} f_{1}^{(1)}(u)+\cos \theta_{2} f_{1}^{(2)}(u)+\cdots+\cos \theta_{p-1} f_{1}^{(p-1)}(u)} .
\end{aligned}
$$

We arrive at (4.9) because $\overline{\boldsymbol{x}}$ corresponds to the defective exponential distribution (4.2).

For $u \geq 0$, we have $r(u) \neq 0$. If $r(u)<0$ then $\overrightarrow{P Q}$ never meets the hyperplane $g(\boldsymbol{x}, u)=0$. However, the ray emanating from $P$ in the direction determined by $\left(\pi-\theta_{1}, \pi-\theta_{2}, \ldots, \pi-\right.$ $\left.\theta_{p-1}\right)$, that is, opposite to $\overrightarrow{P Q}$, does. If this ray meets the hyperplane $g(\boldsymbol{x}, u)=0$ at the point $Y$ (with coordinates $\boldsymbol{y}(u))$, say, then $\|\overrightarrow{P Y}\|_{2}=\|\boldsymbol{y}(u)-\overline{\boldsymbol{x}}\|_{2}=-r(u)$. If $r(u)$ is infinite then $\overrightarrow{P Q}$ is parallel to the hyperplane $g(\boldsymbol{x}, u)=0$.

The vector $\boldsymbol{\theta}$ determined by (4.5) is fixed since $\hat{\boldsymbol{x}}$ is fixed. Using (4.6), define, for $r \in \mathbb{R}$ and $u \geq 0$,

$$
h(r, \boldsymbol{\theta}, u)=g(\boldsymbol{x}(r, \boldsymbol{\theta}), u)=b_{1} f_{1}(u)+\sum_{i=1}^{p-1}\left(\bar{x}_{i}+r \cos \theta_{i}\right) f_{1}^{(i)}(u) .
$$

For any given $u \geq 0$, since $h(r, \boldsymbol{\theta}, u)$ is a linear function of $r$, we have either

$$
\begin{aligned}
& \quad h(r, \boldsymbol{\theta}, u) \geq 0 \text { whenever } r \leq r(u) \\
& \text { and } \quad h(r, \boldsymbol{\theta}, u)<0 \quad \text { whenever } r>r(u),
\end{aligned}
$$

or

$$
\begin{aligned}
& h(r, \boldsymbol{\theta}, u) \geq 0 \quad \text { whenever } r \geq r(u) \\
& \text { and } h(r, \boldsymbol{\theta}, u)<0 \quad \text { whenever } r<r(u) \text {. }
\end{aligned}
$$

Since, for $u \geq 0, h(0, \boldsymbol{\theta}, u)=\lambda_{1} \mathrm{e}^{-\lambda_{1} u}>0$, conditions (4.10) and (4.11) hold when $r(u)>0$, and conditions (4.12) and (4.13) hold when $r(u)<0$.

For $u \geq 0$, we require that

$$
h(r, \boldsymbol{\theta}, u) \geq 0 \quad \text { and } \quad r(u)>0 .
$$

Both inequalities are satisfied when $r \leq \min \{r(u) \mid u \geq 0, r(u)>0\}=r^{*}$. It does not matter if this minimum is attained at more than one value of $u \geq 0$ because it is the quantity $r^{*}$ that we require. If $u^{*}$ is the smallest nonnegative value of $u$ where $r(u)$ attains a minimum when $r(u)>0$, then $r^{*}=r\left(u^{*}\right)$. Let $\hat{r}=\|\hat{\boldsymbol{x}}-\overline{\boldsymbol{x}}\|_{2}$. Now, if $\hat{r} \leq r^{*}$ then the point $Q \in \Omega_{p}$, which implies that $\hat{\boldsymbol{x}} \in \Omega_{p}$, and, consequently, the vectors $\boldsymbol{a}$ and $\boldsymbol{b}$ correspond to an ME distribution. Otherwise, that is, when $\hat{r}>r^{*}$, they do not.

Since $r(u)$ is discontinuous whenever the denominator is equal to zero, instead of finding the minimum of $r(u)$ when $r(u)>0$ over $u \geq 0$, we can equivalently find the global minimum over $u \geq 0$ of the continuous function

$$
\begin{aligned}
d(u) & =-\frac{\lambda_{1}}{r(u)} \\
& =\mathrm{e}^{\lambda_{1} u}\left(\cos \theta_{1} f_{1}^{(1)}(u)+\cos \theta_{2} f_{1}^{(2)}(u)+\cdots+\cos \theta_{p-1} f_{1}^{(p-1)}(u)\right) .
\end{aligned}
$$

If $d^{*}=d\left(u^{*}\right)$ then $r^{*}=-\lambda_{1} / d^{*}$. 
In order to minimize $d(u)$, the inbuilt routine fminbnd in MATLAB ${ }^{\circledR}$, which finds the local minimum of a nonlinear convex function over a finite interval, can be applied repeatedly over intervals where the function is locally convex. In practice, the nature of $d(u)$, determined by the zeros of the polynomial $b(s)$, could be used as a guide in selecting the number and size of such intervals.

According to Reemtsen and Görner [25], however, '... there does not exist an algorithm which is able to detect a global maximizer (or minimizer) of an arbitrary continuous function with certainty.' The ME characterization problem, in general, from an algorithmic point of view, remains a difficult problem to solve. Also, it could be argued that minimizing $d(u)$ is equivalent to minimizing $g(\hat{\boldsymbol{x}}, u)$ and determining if it ever becomes negative. While this is true, the algorithm presented in this section gives a mechanism by which a function of the form (4.1) that does not correspond to an ME distribution can be altered so that it does correspond to an ME distribution. We can achieve this by moving the point $Q$ (which depicts $\hat{\boldsymbol{x}}$, which corresponds to $g(\hat{\boldsymbol{x}}, u)$ ) along $\overline{P Q}$ closer to the point $P$ (which depicts $\overline{\boldsymbol{x}}$, which corresponds to the defective exponential distribution (4.2)). If $\hat{\boldsymbol{x}} \notin \Omega_{p}$ then, for $r \leq r^{*}$,

$$
\boldsymbol{x}=\frac{r}{\hat{r}} \hat{\boldsymbol{x}}+\left(1-\frac{r}{\hat{r}}\right) \overline{\boldsymbol{x}}
$$

will be contained in $\Omega_{p}$ and, hence, will correspond to an ME distribution. Also, as we shall see in Section 6, this approach (that is, minimizing $d(u)$ rather than $g(\boldsymbol{x}, u)$ ) enables a parametric characterization for $\Omega_{3}$.

In Fackrell [14] a semi-infinite programming (SIP) algorithm for determining whether $\hat{\boldsymbol{x}} \in \Omega_{p}$ is presented. In this algorithm $\Omega_{p}$ is approximated by a finite number of constraints, and it may conclude that $\hat{\boldsymbol{x}} \in \Omega_{p}$ when it is in fact not, especially if $\hat{\boldsymbol{x}}$ is near the boundary $\partial \Omega_{p}$. If this is the case, the method presented in this section can be used to refine the answer. The SIP algorithm gives the $u$ values with which to minimize $d(u)$ over, making the ME identification algorithm more effective.

\section{Examples}

In order to illustrate the ME identification method presented in the previous section, and to describe what is happening geometrically, we now consider the following three examples of pairs of vectors $\boldsymbol{a}$ and $\boldsymbol{b}$, and determine if they correspond to ME distributions.

$$
\begin{array}{ll}
\text { 1. } & \boldsymbol{a}=(6,2,2), \quad \boldsymbol{b}=(6,11,6) . \\
\text { 2. } & \boldsymbol{a}=(2.21,-1,1), \quad \boldsymbol{b}=(2.21,4.41,3.2) . \\
\text { 3. } & \boldsymbol{a}=(24,60,60,28,12,1), \quad \boldsymbol{b}=(30,109,159,120,50,11) .
\end{array}
$$

In the first example, since $\lambda_{1}=1, \lambda_{2}=2, \lambda_{3}=3$, and $a_{1}=b_{1}, b(s)$ has zeros -1 , -2 , and -3 , and condition 2 in Section 3 is satisfied. The vectors $\hat{\boldsymbol{x}}$ and $\overline{\boldsymbol{x}}$ are depicted by the points $P(5,1)$ and $Q(2,2)$, respectively; see Figure 3 . We have $\hat{r}=\sqrt{10} \approx 3.16$ and $\cos \boldsymbol{\theta}=\left(\cos \theta_{1}, \cos \theta_{2}\right)=(-3 / \sqrt{10}, 1 / \sqrt{10})$. Figure 3 shows $\Omega_{3}$ with the ray $\overrightarrow{P Q}$ indicated, and Figure 4 shows the accompanying graph of $r(u)$ versus $u$ when $0 \leq u \leq 5$.

We now discuss how $r(u)$ relates to $\Omega_{3}$. For $u \geq 0, r(u)$ is the distance from $P$ to the line $g\left(x_{1}, x_{2}, u\right)=0$ in the direction determined by $\overrightarrow{P Q}$. When $u=0, r(0) \approx-3.16$, which means that the distance $P$ is from the line $g\left(x_{1}, x_{2}, 0\right)=x_{2}=0$ is approximately 3.16 units, but the ray emanating from $P$ that intersects the line is in the opposite direction to $\overrightarrow{P Q}$. The 


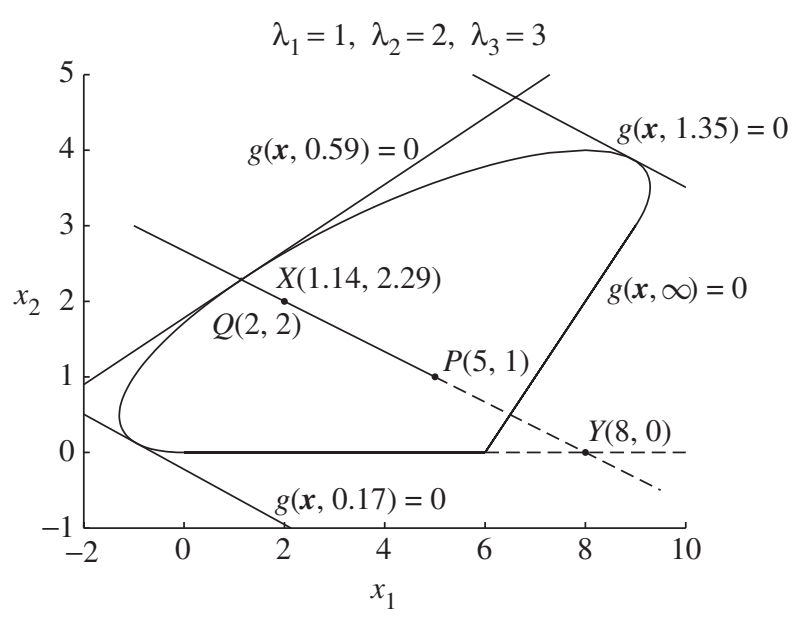

FiguRE 3: Diagram of $\Omega_{3}$ for example 1.

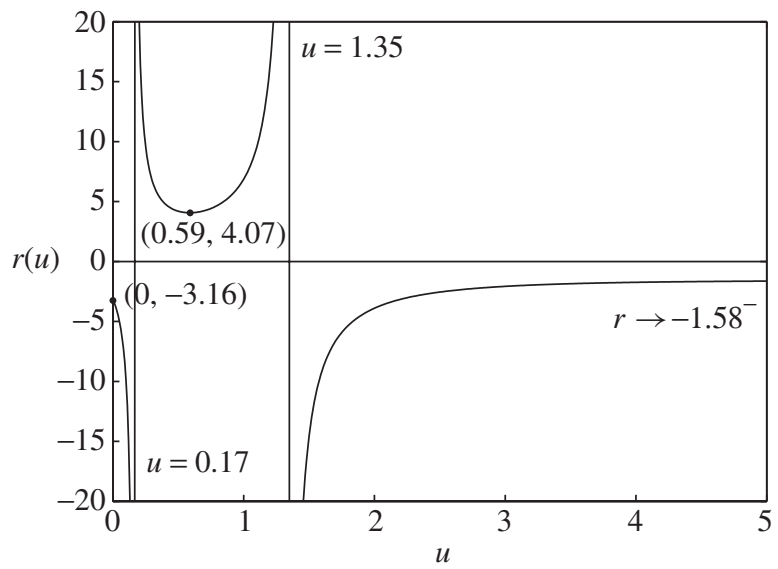

FigURE 4: Graph of $r(u)$ versus $u$ for example 1 .

extension to $\overrightarrow{P Q}$ is depicted in Figure 3 by the dashed line that intersects the line $x_{2}=0$ at the point $Y(8,0)$, and $\|P Y\|_{2} \approx 3.16$. As $u$ increases, $r(u)$ decreases until $u \approx 0.17$, where the corresponding line (plotted on Figure 3 ) is parallel to $\overrightarrow{P Q}$ and $r(u)$ is infinite. This corresponds to the vertical asymptote at $u \approx 0.17$ on the graph of $r(u)$ versus $u$; see Figure 4 . As $u$ increases further, $r(u)$ decreases to a local minimum value of approximately 4.07 when $u \approx 0.59$. This value is the minimum value of $r(u)$ over $u \geq 0$ when $r(u)>0$, that is, $r^{*}$. Thus, $u^{*} \approx 0.59$ and $r^{*} \approx 4.07$. The value of $r^{*}$ is the maximum distance a point $X\left(x_{1}, x_{2}\right)$ can be from $P$ in the direction of $\overrightarrow{P Q}$ such that it is in $\Omega_{3}$ (or such that $g\left(x_{1}, x_{2}, u\right) \geq 0$ for all $u \geq 0$ ). The point $X$ that is $r^{*}$ units from $P$ occurs at the intersection of $\overrightarrow{P Q}$ and the line $g\left(x_{1}, x_{2}, u^{*}\right)=0$, and is indicated in Figure 3. The coordinates of $X$ are approximately $(1.14,2.29)$. Since $\hat{r}<r^{*}$, the point $Q$ (that is, $\hat{\boldsymbol{x}}$ ) is contained in $\Omega_{3}$ and the vectors given in (5.1) correspond to an $\mathrm{ME}$ 
distribution. As $u$ increases from $u^{*}, r(u)$ increases to $\infty$ when $u \approx 1.35$. This corresponds to the vertical asymptote at $u \approx 1.35$ in Figure 4 . The corresponding line, which is parallel to $\overrightarrow{P Q}$, is plotted in Figure 3. For $u \gtrsim 1.35, r(u)$ is always negative and $\lim _{u \rightarrow \infty} r(u) \approx-1.58$. Thus, as $u \rightarrow \infty, P$ is approximately 1.58 units from the line $g\left(x_{1}, x_{2}, u\right)=0$ in the direction opposite to $\overrightarrow{P Q}$. In this example, to minimize $d(u)$, we used the fminbnd function in MATLAB repeatedly over $[0,2]$ in intervals of length 0.1 . The run time was approximately 3 seconds.

In the second example, $\lambda_{1}=1, \lambda_{2}=1.1+\mathrm{i}, \lambda_{3}=1.1-\mathrm{i}$, and $a_{1}=b_{1}$. Thus, the zero of $b(s)$ of maximal real part is real and negative, and condition 2 in Section 3 is satisfied. Here, $\overline{\boldsymbol{x}}=(2.21,1)$ (the coordinates of $P$ ) and $\hat{\boldsymbol{x}}=(-1,1)$ (the coordinates of $Q$ ). We have $\hat{r} \approx 3.20$ and $\cos \theta=\left(\cos \theta_{1}, \cos \theta_{2}\right)=(-1,0)$. For this example, $r^{*} \approx 2.65$, which occurs when $u^{*} \approx 0.74$. The ray $\overrightarrow{P Q}$ meets the hyperplane $g\left(x_{1}, x_{2}, 0.74\right)=0$ at the point $X(-0.44,1)$. Since $\hat{r}>r^{*}$, the point $Q$ (and, hence, $\hat{\boldsymbol{x}}$ ) is not contained in $\Omega_{3}$ and so the vectors given in (5.2) do not correspond to an ME distribution. From (4.14), for $r \lesssim 2.65$, any vector of the form

$$
\boldsymbol{x}=\frac{r}{3.20}(-1,1)+\left(1-\frac{r}{3.20}\right)(2.21,1)=\left(2.21-\frac{3.21}{3.2} r, 1\right)
$$

corresponds to an ME distribution. For this example, $d(u)$ was minimized over [0, 2] in intervals of length 0.1 . The run time was approximately 3.5 seconds. A detailed description of how $\Omega_{3}$ relates to the graph of $r(u)$, as we gave for example 1, can be found in [12, Section 7.4].

In the final example, $\lambda_{1}=1, \lambda_{2}=1, \lambda_{3}=2, \lambda_{4}=2+\mathrm{i}, \lambda_{5}=2-\mathrm{i}, \lambda_{6}=3$, and $a_{1}=\frac{4}{5} b_{1}$. Thus, the zero of maximal real part of $b(s)$ is real and negative, and condition 2 in Section 3 is satisfied. The vectors $\overline{\boldsymbol{x}}$ and $\hat{\boldsymbol{x}}$ (corresponding to points $P$ and $Q$, respectively) are $(79,80,40,10,1)$ and $(75,75,35,15,1.25)$, respectively. We have $\hat{r} \approx 9.5427$ and $\left(\cos \theta_{1}, \cos \theta_{2}, \cos \theta_{3}, \cos \theta_{4}, \cos \theta_{5}\right) \approx(-0.4192,-0.5240,-0.5240,0.5240,0.0262)$. The plot of $r(u)$ versus $u$ is shown in Figure 5, where we can see that $r^{*} \approx 10.05$ when $u^{*} \approx 4.31$. Since $\hat{r}<r^{*}$, the vectors given in (5.3) correspond to an ME distribution. The graph of $u$ versus $r(u)$ gives us the distance the point $P$ is from the hyperplane $g(\boldsymbol{x}, u)=0$ and whether or not

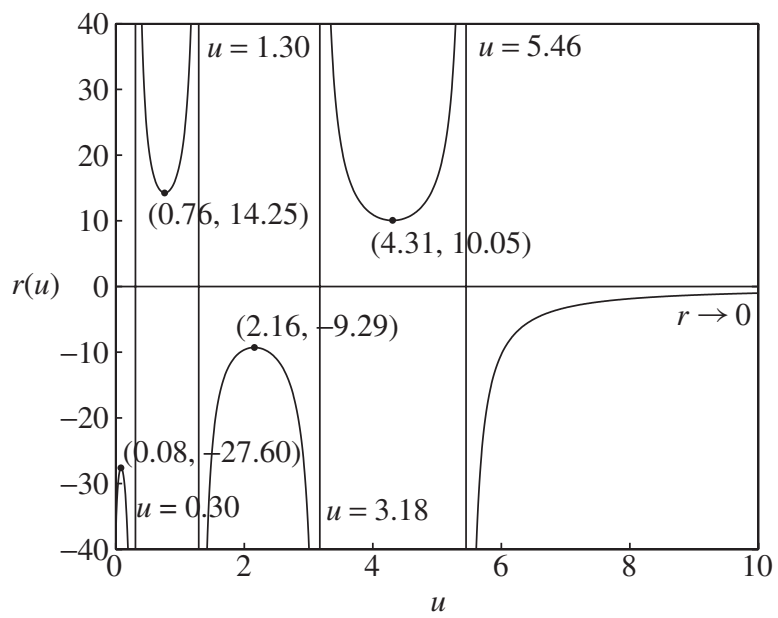

FIGURE 5: Graph of $r(u)$ versus $u$ for example 3. 
the intersection of the line $\overline{P Q}$ with the hyperplane is in the same direction as $\overrightarrow{P Q}$. The graph suggests that the geometry of the situation is quite complicated. For this example, $d(u)$ was minimized over $[0,5]$ in intervals of length 0.1 . The run time was approximately 9.5 seconds.

The SIP algorithm mentioned at the end of Section 4 took 0.1 seconds to converge for an order 3 example, and 0.2 seconds for an order 5 example. But, when the solution was refined using the method described in the previous section, the run times were comparable. In this case, the set of $u$ values that $d(u)$ was minimized over was significantly reduced because the SIP algorithm gave a good indication of where the solution was located.

\section{Another parameterization of $\boldsymbol{\Omega}_{3}$}

In this section, using the method developed in Section 4, we give an alternative parameterization of $\partial \Omega_{3}$ to those given in Theorems 3.1 and 3.2. Theorem 6.1, below, gives the parameterization when the zeros of the polynomial $b(s)$ are real and distinct. There are similar results when some or all of the zeros are repeated, but we do not state them here. They can be found in [12, Theorem 7.3]. Theorem 6.2, below, gives the parameterization when two of the zeros are a complex conjugate pair and is stated without proof. The proof can be found in [12, Theorem 7.4].

Theorem 6.1. Suppose that the vectors $\boldsymbol{a}=\left(a_{1}, a_{2}, a_{3}\right)$ and $\boldsymbol{b}=\left(b_{1}, b_{2}, b_{3}\right)$ are such that $0<a_{1} / b_{1} \leq 1$, and the zeros of $b(s)=s^{3}+b_{3} s^{2}+b_{2} s+b_{1},-\lambda_{3},-\lambda_{2}$, and $-\lambda_{1}$, are real and such that $0<\lambda_{1}<\lambda_{2}<\lambda_{3}$. If $\theta_{1}, \theta_{2}$, and $\theta_{3}$ are such that

$$
\begin{gathered}
\tan \theta_{1}=\frac{1}{\lambda_{2}+\lambda_{3}} \text { and } \pi<\theta_{1}<\frac{3 \pi}{2}, \\
\tan \theta_{2}=\frac{\lambda_{1}}{\lambda_{1} \lambda_{3}+\lambda_{1} \lambda_{2}-\lambda_{2} \lambda_{3}} \text { and } \pi<\theta_{2}<2 \pi, \\
\text { and } \tan \theta_{3}=\frac{1}{\lambda_{2}} \text { and } 0<\theta_{3}<\frac{\pi}{2},
\end{gathered}
$$

then the parametric representation for $\partial \Omega_{3}$ is as follows.

1. For $\theta_{1}<\theta \leq \theta_{2}$,

$$
\begin{aligned}
& x_{1}(\theta)=-\lambda_{1} \cot \theta+\lambda_{1}\left(\lambda_{2}+\lambda_{3}\right), \\
& x_{2}(\theta)=0 .
\end{aligned}
$$

2. For $\theta_{2}<\theta \leq \theta_{3}+2 \pi$,

$$
\begin{aligned}
& x_{1}(\theta)=\frac{\left(\lambda_{1}^{2}+\lambda_{2} \lambda_{3}\right) \cos \theta-\lambda_{1}^{2}\left(\lambda_{2}+\lambda_{3}\right) \sin \theta}{\cos \theta-\lambda_{1} \sin \theta}, \\
& x_{2}(\theta)=\frac{\lambda_{1} \cos \theta+\left(\lambda_{2} \lambda_{3}-\lambda_{1} \lambda_{3}-\lambda_{1} \lambda_{2}\right) \sin \theta}{\cos \theta-\lambda_{1} \sin \theta} .
\end{aligned}
$$

3. For $\theta_{3}<\theta \leq \theta_{1}$,

$$
\begin{aligned}
x_{1}(\theta)= & \frac{\lambda_{1}\left(\lambda_{3}-\lambda_{1}\right)\left(\lambda_{2}-\lambda_{1}\right) \cos \theta}{\lambda_{1}\left(\cos \theta-\lambda_{1} \sin \theta\right)-\lambda_{3}\left(\cos \theta-\lambda_{3} \sin \theta\right) \gamma(\theta)^{\left(\lambda_{1}-\lambda_{3}\right) /\left(\lambda_{3}-\lambda_{2}\right)}} \\
& +\lambda_{1}\left(\lambda_{2}+\lambda_{3}\right), \\
x_{2}(\theta)= & \frac{\lambda_{1}\left(\lambda_{3}-\lambda_{1}\right)\left(\lambda_{2}-\lambda_{1}\right) \sin \theta}{\lambda_{1}\left(\cos \theta-\lambda_{1} \sin \theta\right)-\lambda_{3}\left(\cos \theta-\lambda_{3} \sin \theta\right) \gamma(\theta)^{\left(\lambda_{1}-\lambda_{3}\right) /\left(\lambda_{3}-\lambda_{2}\right)}}+\lambda_{1},
\end{aligned}
$$




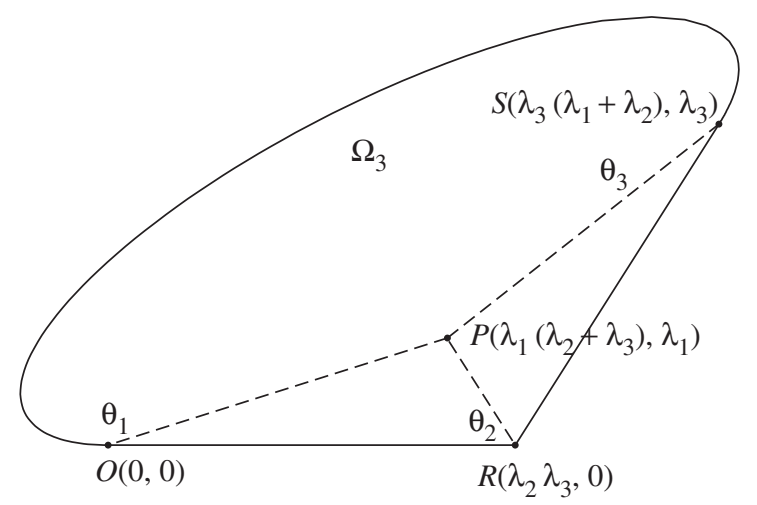

FIGURE 6: Diagram of $\Omega_{3}$ showing the points $O, P, R$, and $S$.

where

$$
\gamma(\theta)=\frac{\lambda_{3}\left(\cos \theta-\lambda_{3} \sin \theta\right)}{\lambda_{2}\left(\cos \theta-\lambda_{2} \sin \theta\right)} .
$$

Proof. Refer to Figure 6 for a diagram of the situation. Recall from Theorem 3.1 that $\partial \Omega_{3}$ consists of

1. the line segment between $O(0,0)$ and $R\left(\lambda_{2} \lambda_{3}, 0\right)$,

2. the line segment between $R$ and $S\left(\lambda_{3}\left(\lambda_{2}+\lambda_{1}\right), \lambda_{3}\right)$, and

3. the parametric curve $\Sigma_{3}$ which has as its endpoints $O$ and $S$.

We show that these three sections of $\partial \Omega_{3}$ are parameterized by (6.1) and (6.2), (6.3) and (6.4), and (6.5) and (6.6), respectively.

Recall, also, that the mixture of the point mass at zero and the exponential distribution (4.2) is depicted in Figure 6 by the point $P\left(\lambda_{1}\left(\lambda_{2}+\lambda_{3}\right), \lambda_{1}\right)$; see (4.3) and (4.4). Let $\theta$ be the angle between the ray emanating from $P$ parallel to the positive $x_{1}$ axis and any ray emanating from $P$, measured in an anticlockwise direction. Let $\theta_{1}, \theta_{2}$, and $\theta_{3}$ be such angles that are defined by the rays $\overrightarrow{P O}, \overrightarrow{P R}$, and $\overrightarrow{P S}$, respectively. We have $\tan \theta_{1}=1 /\left(\lambda_{2}+\lambda_{3}\right)$, and since the coordinates of $P$ are both positive, then $\pi<\theta_{1}<3 \pi / 2$. Also, $\tan \theta_{2}=\lambda_{1} /\left(\lambda_{1} \lambda_{3}+\lambda_{1} \lambda_{2}-\lambda_{2} \lambda_{3}\right)$, and since the $x_{2}$ coordinate of $P$ is positive, then $\pi<\theta_{2}<2 \pi$. Lastly, $\tan \theta_{3}=1 / \lambda_{2}$, and since both coordinates of $S$ are greater than their respective coordinates of $P$, then $0<\theta_{3}<\pi / 2$.

For $p=3$, from (4.9),

$$
r(u)=\frac{-\lambda_{1} \mathrm{e}^{-\lambda_{1} u}}{\cos \theta f_{1}^{(1)}(u)+\sin \theta f_{1}^{(2)}(u)},
$$

and, therefore,

$$
d(u)=-\frac{\lambda_{1}}{r(u)}=\mathrm{e}^{\lambda_{1} u}\left(\cos \theta f_{1}^{(1)}(u)+\sin \theta f_{1}^{(2)}(u)\right) .
$$

Here, we note that, when the zeros of $b(s)$ are distinct,

$$
f_{1}(u)=\frac{\mathrm{e}^{-\lambda_{1} u}}{\left(\lambda_{1}-\lambda_{2}\right)\left(\lambda_{1}-\lambda_{3}\right)}+\frac{\mathrm{e}^{-\lambda_{2} u}}{\left(\lambda_{2}-\lambda_{1}\right)\left(\lambda_{2}-\lambda_{3}\right)}+\frac{\mathrm{e}^{-\lambda_{3} u}}{\left(\lambda_{3}-\lambda_{1}\right)\left(\lambda_{3}-\lambda_{2}\right)} .
$$


The function $d(u)$ attains a global minimum when one of the following three situations occurs:

1. $u=0$,

2. $u \rightarrow \infty$, or

3. $d^{\prime}(u)=0$.

Suppose that the global minimum is achieved when $u=u^{*}=0$. The minimum value of $d(u)$ is therefore

$$
d^{*}=d(0)=\sin \theta .
$$

Thus, the corresponding points on the boundary $\partial \Omega_{3}$, using (4.6), are given by

$$
x_{1}(\theta)=-\frac{\lambda_{1}}{d^{*}} \cos \theta+\lambda_{1}\left(\lambda_{2}+\lambda_{3}\right)=-\lambda_{1} \cot \theta+\lambda_{1}\left(\lambda_{2}+\lambda_{3}\right)
$$

and

$$
x_{2}(\theta)=-\frac{\lambda_{1}}{d^{*}} \sin \theta+\lambda_{1}=0 .
$$

This situation occurs when the ray emanating from $P$ in the direction defined by $\theta$ intersects the line $g\left(x_{1}, x_{2}, 0\right)=0$, that is, $\overleftrightarrow{O R}$. Thus, we have $\theta_{1}<\theta \leq \theta_{2}$. Note that (6.9) is defined for these values of $\theta$ because $\cot \theta$ is defined for $\pi<\theta<2 \pi$.

Suppose that the global minimum is achieved as $u \rightarrow \infty$. The minimum value is therefore

$$
\begin{aligned}
d^{*} & =\lim _{u \rightarrow \infty} \mathrm{d}(u) \\
& =\lim _{u \rightarrow \infty} \mathrm{e}^{\lambda_{1} u}\left(\cos \theta f_{1}^{(1)}(u)+\sin \theta f_{1}^{(2)}(u)\right) \\
& =\frac{-\lambda_{1} \cos \theta+\lambda_{1}^{2} \sin \theta}{\left(\lambda_{3}-\lambda_{1}\right)\left(\lambda_{2}-\lambda_{1}\right)} .
\end{aligned}
$$

The last equality is achieved by observing, from (6.8), that the dominant terms in $f_{1}^{(1)}(u)$ and $f_{1}^{(2)}(u)$ as $u \rightarrow \infty$ are

$$
\frac{-\lambda_{1} \mathrm{e}^{-\lambda_{1} u}}{\left(\lambda_{1}-\lambda_{3}\right)\left(\lambda_{1}-\lambda_{2}\right)} \text { and } \frac{\lambda_{1}^{2} \mathrm{e}^{-\lambda_{1} u}}{\left(\lambda_{1}-\lambda_{2}\right)\left(\lambda_{1}-\lambda_{3}\right)},
$$

respectively. Thus, the points on the boundary $\partial \Omega_{3}$ are given by

$$
\begin{aligned}
x_{1}(\theta) & =-\frac{\lambda_{1}}{d^{*}} \cos \theta+\lambda_{1}\left(\lambda_{2}+\lambda_{3}\right) \\
& =\frac{\left(\lambda_{3}-\lambda_{1}\right)\left(\lambda_{2}-\lambda_{1}\right) \cos \theta}{\cos \theta-\lambda_{1} \sin \theta}+\lambda_{1}\left(\lambda_{2}+\lambda_{3}\right) \\
& =\frac{\left(\lambda_{1}^{2}+\lambda_{2} \lambda_{3}\right) \cos \theta-\lambda_{1}^{2}\left(\lambda_{2}+\lambda_{3}\right) \sin \theta}{\cos \theta-\lambda_{1} \sin \theta}
\end{aligned}
$$

and

$$
\begin{aligned}
x_{2}(\theta) & =-\frac{\lambda_{1}}{d^{*}} \sin \theta+\lambda_{1} \\
& =\frac{\left(\lambda_{3}-\lambda_{1}\right)\left(\lambda_{2}-\lambda_{1}\right) \sin \theta}{\cos \theta-\lambda_{1} \sin \theta}+\lambda_{1} \\
& =\frac{\lambda_{1} \cos \theta+\left(\lambda_{2} \lambda_{3}-\lambda_{1} \lambda_{3}-\lambda_{1} \lambda_{2}\right) \sin \theta}{\cos \theta-\lambda_{1} \sin \theta} .
\end{aligned}
$$


This occurs when the ray emanating from $P$ in the direction defined by $\theta$ intersects the line $g\left(x_{1}, x_{2}, u\right)=0$ as $u \rightarrow \infty$, that is, $\overleftrightarrow{R S}$. Thus, $\theta_{2}<\theta \leq 2 \pi+\theta_{3}$. Note that (6.10) and (6.11) are defined for such $\theta$ since $\cos \theta-\lambda_{1} \sin \theta>0$ (see [12, p. 136]).

Suppose that the global minimum is achieved when $d^{\prime}(u)=0$. We have, using expression (6.8) for $f_{1}(u)$,

$$
\begin{aligned}
d^{\prime}(u) & =\mathrm{e}^{\lambda_{1} u}\left(\lambda_{1} \cos \theta f_{1}^{(1)}(u)+\left(\cos \theta+\lambda_{1} \sin \theta\right) f_{1}^{(2)}(u)+\sin \theta f_{1}^{(3)}\right) \\
& =\mathrm{e}^{\lambda_{1} u} \frac{\left(\cos \theta-\lambda_{3} \sin \theta\right) \lambda_{3} \mathrm{e}^{-\lambda_{3} u}-\left(\cos \theta-\lambda_{2} \sin \theta\right) \lambda_{2} \mathrm{e}^{-\lambda_{2} u}}{\lambda_{3}-\lambda_{2}} .
\end{aligned}
$$

Solving $d^{\prime}(u)=0$ and letting the value of $u$ when this occurs be $u^{*}=u^{*}(\theta)$ gives

$$
\mathrm{e}^{u^{*}(\theta)}=\left(\frac{\lambda_{3}\left(\cos \theta-\lambda_{3} \sin \theta\right)}{\lambda_{2}\left(\cos \theta-\lambda_{2} \sin \theta\right)}\right)^{1 /\left(\lambda_{3}-\lambda_{2}\right)} .
$$

The function $d(u)$ attains a minimum when $u=u^{*}$. This is the case because

$$
\begin{aligned}
d^{\prime \prime}\left(u^{*}\right)= & \frac{\mathrm{e}^{\lambda_{1} u^{*}}}{\lambda_{3}-\lambda_{2}}\left(\left(\cos \theta-\lambda_{3} \sin \theta\right) \lambda_{1} \lambda_{3} \mathrm{e}^{-\lambda_{3} u^{*}}-\left(\cos \theta-\lambda_{2} \sin \theta\right) \lambda_{1} \lambda_{2} \mathrm{e}^{-\lambda_{2} u^{*}}\right. \\
& \left.-\left(\cos \theta-\lambda_{3} \sin \theta\right) \lambda_{3}^{2} \mathrm{e}^{-\lambda_{3} u^{*}}+\left(\cos \theta-\lambda_{2} \sin \theta\right) \lambda_{2}^{2} \mathrm{e}^{-\lambda_{2} u^{*}}\right) \\
= & \frac{\mathrm{e}^{\lambda_{1} u^{*}}}{\lambda_{3}-\lambda_{2}}\left(-\left(\cos \theta-\lambda_{3} \sin \theta\right) \lambda_{3}^{2} \mathrm{e}^{-\lambda_{3} u^{*}}+\left(\cos \theta-\lambda_{2} \sin \theta\right) \lambda_{2}^{2} \mathrm{e}^{-\lambda_{2} u^{*}}\right) \\
> & \frac{\mathrm{e}^{\lambda_{1} u^{*}}}{\lambda_{3}-\lambda_{2}}\left(-\left(\cos \theta-\lambda_{3} \sin \theta\right) \lambda_{3}^{2} \mathrm{e}^{-\lambda_{3} u^{*}}+\left(\cos \theta-\lambda_{2} \sin \theta\right) \lambda_{2} \lambda_{3} \mathrm{e}^{-\lambda_{2} u^{*}}\right) \\
= & 0 .
\end{aligned}
$$

The second and third equalities are due to the fact that $d^{\prime}\left(u^{*}\right)=0$ (see (6.12)), and the inequality is because $0<\lambda_{2}<\lambda_{3}$, and, for $\theta_{3}<\theta \leq \theta_{1}, \cos \theta-\lambda_{2} \sin \theta<0$ (see [12, pp. 137-138]).

Define, for $\theta_{3}<\theta \leq \theta_{1}$,

$$
\gamma(\theta)=\frac{\lambda_{3}\left(\cos \theta-\lambda_{3} \sin \theta\right)}{\lambda_{2}\left(\cos \theta-\lambda_{2} \sin \theta\right)} .
$$

For $\theta_{3}<\theta \leq \theta_{1},(6.13)$ is defined since $\gamma(\theta)>0$ (see [12, pp. 137-138]).

Equating the expression for $d^{\prime}\left(u^{*}\right)$ in (6.12) to zero we have

$$
\lambda_{2} \mathrm{e}^{-\lambda_{2} u^{*}(\theta)}\left(\cos \theta-\lambda_{2} \sin \theta\right)=\lambda_{3} \mathrm{e}^{-\lambda_{3} u^{*}(\theta)}\left(\cos \theta-\lambda_{3} \sin \theta\right) .
$$

Substituting this expression into (6.7), after some rearrangement, gives

$$
\begin{aligned}
d\left(u^{*}(\theta)\right) & =-\frac{\lambda_{1}\left(\cos \theta-\lambda_{1} \sin \theta\right)-\lambda_{3}\left(\cos \theta-\lambda_{3} \sin \theta\right) \mathrm{e}^{\left(\lambda_{1}-\lambda_{3}\right) u^{*}(\theta)}}{\left(\lambda_{3}-\lambda_{1}\right)\left(\lambda_{2}-\lambda_{1}\right)} \\
& =-\frac{\lambda_{1}\left(\cos \theta-\lambda_{1} \sin \theta\right)-\lambda_{3}\left(\cos \theta-\lambda_{3} \sin \theta\right) \gamma(\theta)^{\left(\lambda_{1}-\lambda_{3}\right) /\left(\lambda_{3}-\lambda_{2}\right)}}{\left(\lambda_{3}-\lambda_{1}\right)\left(\lambda_{2}-\lambda_{1}\right)},
\end{aligned}
$$

by (6.13). Thus, the points on $\partial \Omega_{3}$ are given by

$$
\begin{aligned}
x_{1}(\theta) & =-\frac{\lambda_{1}}{d^{*}} \cos \theta+\lambda_{1}\left(\lambda_{2}+\lambda_{3}\right) \\
& =\frac{\lambda_{1}\left(\lambda_{3}-\lambda_{1}\right)\left(\lambda_{2}-\lambda_{1}\right) \cos \theta}{\lambda_{1}\left(\cos \theta-\lambda_{1} \sin \theta\right)-\lambda_{3}\left(\cos \theta-\lambda_{3} \sin \theta\right) \gamma(\theta)^{\left(\lambda_{1}-\lambda_{3}\right) /\left(\lambda_{3}-\lambda_{2}\right)}}+\lambda_{1}\left(\lambda_{2}+\lambda_{3}\right)
\end{aligned}
$$


and

$$
\begin{aligned}
x_{2}(\theta) & =-\frac{\lambda_{1}}{d^{*}} \sin \theta+\lambda_{1} \\
& =\frac{\lambda_{1}\left(\lambda_{3}-\lambda_{1}\right)\left(\lambda_{2}-\lambda_{1}\right) \sin \theta}{\lambda_{1}\left(\cos \theta-\lambda_{1} \sin \theta\right)-\lambda_{3}\left(\cos \theta-\lambda_{3} \sin \theta\right) \gamma(\theta)^{\left(\lambda_{1}-\lambda_{3}\right) /\left(\lambda_{3}-\lambda_{2}\right)}}+\lambda_{1} .
\end{aligned}
$$

This occurs when the ray emanating from $P$ in the direction defined by $\theta$ intersects the line $g\left(x_{1}, x_{2}, u\right)=0$, where $u>0$. Thus, $\theta_{3}<\theta \leq \theta_{1}$.

We now give an alternative parameterization of $\partial \Omega_{3}$ when two of the zeros of $b(s)$ are a complex conjugate pair.

Theorem 6.2. Suppose that the vectors $\boldsymbol{a}=\left(a_{1}, a_{2}, a_{3}\right)$ and $\boldsymbol{b}=\left(b_{1}, b_{2}, b_{3}\right)$ are such that $0<a_{1} / b_{1} \leq 1$, and the zeros of $b(s)=s^{3}+b_{3} s^{2}+b_{2} s+b_{1},-\lambda_{1},-\lambda_{2}$, and $-\lambda_{3}$, are such that $\lambda_{1}=\mu, \lambda_{2}=\alpha+\mathrm{i} \beta$, and $\lambda_{3}=\alpha+\mathrm{i} \beta$ with $0<\mu \leq \alpha$ and $\beta>0$. Let $f_{1}(u)$ be defined by (3.1). If $\theta_{1}$ and $\theta_{2}$ are such that

$$
\begin{gathered}
\tan \theta_{1}=\frac{1}{2 \alpha} \text { and } \pi<\theta_{1}<\frac{3 \pi}{2}, \\
\text { and } \tan \theta_{2}=\frac{\mu}{2 \mu \alpha-x_{1}(\tilde{u})},
\end{gathered}
$$

where $\tilde{u}$ is the minimal positive solution to

$$
f_{1}(u) f_{1}^{(2)}(u)-\left(f_{1}^{(1)}(u)\right)^{2}=0,
$$

and

$$
x_{1}(\tilde{u})=-b_{1} \frac{f_{1}(\tilde{u})}{f_{1}^{(1)}\left(u^{*}\right)},
$$

and $\pi<\theta_{2}<2 \pi$, then the parametric representation for $\partial \Omega_{3}$ is as follows.

1. For $\theta_{1} \leq \theta<\theta_{2}$,

$$
x_{1}(\theta)=-\mu \cot \theta+2 \mu \alpha, \quad x_{2}(\theta)=0 .
$$

2. For $\theta_{2} \leq \theta<2 \pi+\theta_{1}$,

$$
\begin{aligned}
& x_{1}(\theta)=\frac{\mu\left((\mu-\alpha)^{2}+\beta^{2}\right) \cos \theta}{\mu(\cos \theta-\mu \sin \theta)-w(\theta)^{z} \overline{w(\theta)} \bar{z}}+2 \mu \alpha, \\
& x_{2}(\theta)=\frac{\mu\left((\mu-\alpha)^{2}+\beta^{2}\right) \sin \theta}{\mu(\cos \theta-\mu \sin \theta)-w(\theta)^{z} \overline{w(\theta)} \bar{z}}+\mu,
\end{aligned}
$$

where

$$
w(\theta)=\alpha \cos \theta-\left(\alpha^{2}-\beta^{2}\right) \sin \theta+\mathrm{i}(\beta \cos \theta-2 \alpha \beta \sin \theta)
$$

and

$$
z=\frac{1}{2}+\mathrm{i} \frac{\alpha-\mu}{2 \beta} .
$$

The expressions $\overline{w(\theta)}$ and $\bar{z}$ denote the complex conjugates of $w(\theta)$ and $z$, respectively. 


\section{Conclusion}

In this paper we have reduced the problem of determining whether or not the vectors $\boldsymbol{a}$ and $\boldsymbol{b}$ correspond to an ME distribution to one of finding the global minimum of a continuous single-variable function $d(u)$ over the nonnegative real numbers. While, as mentioned in the penultimate paragraph of Section 4 , this is not necessarily a straightforward exercise. If the size of $\boldsymbol{a}$ and $\boldsymbol{b}$ is relatively low, the optimization can be performed accurately within a reasonable time. A problem would arise, however, even for the low-order case, if many applications of the algorithm are required because of the time such a task would take. This problem would arise when fitting ME distributions to data because the algorithm would need to be used each time the parameter values are updated.

When $p=3$, though, the function $d(u)$ has a unique minimum (when it exists) on $[0, \infty$ ) and as a result an alternative parametric description of $\Omega_{3}$ is found. The method used in this paper to give a complete description of $\Omega_{3}$ ought to be able to be applied, at least to some extent, in developing a description of $\Omega_{p}$ when $p>3$. The main problem with describing $\Omega_{p}$ is that the hyperplanes (4.7) are parameterized by a single real variable $u$, and so the envelope (intersection of all the hyperplanes) forms a one-dimensional curve in $\mathbb{R}^{p-1}$, which, unless $p=3$, cannot enclose a $(p-1)$-dimensional region. However, the author believes that he has a description of $\Omega_{4}$ for most configurations of the zeros of $b(s)$. This is the focus of ongoing work.

The SIP algorithm in [14], mentioned at the end of Section 4, is quite efficient, and, if necessary, the corresponding $d(u)$ need only be minimized over a small interval to obtain an accurate solution to the problem of determining if $\boldsymbol{a}$ and $\boldsymbol{b}$ correspond to an ME distribution. In fact, the SIP approach was used in an algorithm to fit ME distributions to data using maximum likelihood in [13]. Here, the final solution was refined using the method described in Section 4.

\section{References}

[1] Asmussen, S. (2000). Ruin Probabilities (Adv. Ser. Statist. Sci. 2), World Scientific, River Edge, NJ.

[2] Asmussen, S. and Bladt, M. (1997). Renewal theory and queueing algorithms for matrix-exponential distributions. In Matrix-Analytic Methods in Stochastic Models (Lecture Notes Pure Appl. Math. 183), eds S. R. Chakravarthy and A. S. Alfa, Dekker, New York, pp. 313-341.

[3] Asmussen, S. And Bladt, M. (1999). Point processes with finite-dimensional conditional probabilities. Stoch. Process. Appl. 82, 127-142.

[4] Asmussen, S. and O'Cinneide, C. A. (1997). Matrix-exponential distributions-distributions with a rational Laplace transform. In Encyclopedia of Statistical Sciences, eds S. Kotz and C. B. Read, John Wiley, New York, pp. $435-440$.

[5] Asmussen, S. ANd Rolski, T. (1992). Computational methods in risk theory: a matrix-algorithmic approach. Insurance Math. Econom. 10, 259-274.

[6] Bean, N. G., Fackrell, M. and Taylor, P. (2008). Characterization of matrix-exponential distributions. Stoch. Models 24, 339-363.

[7] Bladt, M. ANd Neuts, M. F. (2003). Matrix-exponential distributions: calculus and interpretations via flows. Stoch. Models 19, 113-124.

[8] Cohen, J. W. (1969). The Single Server Queue (North-Holland Ser. Appl. Math. Mech. 8). North-Holland, Amsterdam.

[9] Cox, D. R. (1955). A use of complex probabilities in the theory of stochastic processes. Proc. Camb. Phil. Soc. 51, 313-319.

[10] Cox, D. R. (1955). The analysis of non-Markovian stochastic processes by the inclusion of supplementary variables. Proc. Camb. Phil. Soc. 51, 433-441.

[11] Dehon, M. and Latouche, G. (1982). A geometric interpretation of the relations between the exponential and generalized Erlang distributions. Adv. Appl. Prob. 14, 885-897.

[12] Fackrell, M. (2003). Characterization of matrix-exponential distributions. Doctoral Thesis, University of Adelaide. Available at http://thesis.library.adelaide.edu.au/public/adt-SUA20051207.123257/.

[13] FACKRELl, M. (2005). Fitting with matrix-exponential distributions. Stoch. Models 21, 377-400. 
[14] FACKRELL, M. (2008). A semi-infinite programming approach to characterizing matrix exponential distributions. Submitted.

[15] Harris, C. M., Marchal, W. G. and Botta, R. B. (1992). A note on generalized hyperexponential distributions. Commun. Statist. Stoch. Models 8, 179-191.

[16] Lee, Y. D., VAn de Liefvoort, A. And Wallace, V. L. (2000). Modeling correlated traffic with a generalized IPP. Performance Evaluation 40, 99-114.

[17] LIPSKY, L. (1992). Queueing Theory: A Linear Algebraic Approach. Macmillan, New York.

[18] Lipsky, L. AND RAMASwami, V. (1985). A unique minimal representation of Coxian service centres. Tech. Rep., Department of Computer Science and Engineering, University of Nebraska.

[19] Medhi, D., Van de Liefvoort, A. and Reece, C. S. (1995). Performance analysis of a digital link with heterogeneous multislot traffic. IEEE Trans. Commun. 43, 968-976.

[20] Neuts, M. F. (1975). Probability distributions of phase-type. In Liber Amicorum Prof. Emeritus H. Florin, University of Louvain, pp. 173-206.

[21] Neuts, M. F. (1981). Matrix-Geometric Solutions in Stochastic Models. Johns Hopkins University Press, Baltimore.

[22] O’Cinneide, C. A. (1990). Characterization of phase-type distributions. Commun. Statist. Stoch. Models 6, $1-57$.

[23] O’Cinneide, C. A. (1991). Phase-type distributions and invariant polytopes. Adv. Appl. Prob. 23, 515-535.

[24] O'Cinneide, C. A. (1993). Triangular order of triangular phase-type distributions. Commun. Statist. Stoch. Models 9, 507-529.

[25] Reemtsen, R. And Görner, S. (1998). Numerical methods for semi-infinite programming: a survey. In SemiInfinite Programming (Nonconvex Optim. Appl. 25), eds R. Reemtsen and J.-J. Rückmann, Kluwer, Boston, MA, pp. 195-275.

[26] Smith, W. L. (1953). On the distribution of queueing times. Proc. Camb. Phil. Soc. 49, 449-461.

[27] Sumita, U. AND Masuda, Y. (1987). Classes of probability density functions having Laplace transforms with negative zeros and poles. Adv. Appl. Prob. 19, 632-651.

[28] Zemanian, A. H. (1959). On the pole and zero locations of rational Laplace transformations of non-negative functions. Proc. Amer. Math. Soc. 10, 868-872.

[29] Zemanian, A. H. (1961). On the pole and zero locations of rational Laplace transformations of non-negative functions. II. Proc. Amer. Math. Soc. 12, 870-874. 